\title{
Method Research of Coal Mine Roof Strain Detection Based on the Fiber Bragg Grating Sensor with Thermodynamic Couple Numerical Condition
}

\author{
Zhang Jian, ${ }^{1}$ Lu Chuan, ${ }^{2}$ and Lin De-Shu ${ }^{1}$ \\ ${ }^{1}$ College of Computer Science, Yangtze University, Hubei, Jingzhou 434023, China \\ ${ }^{2}$ Liberal Arts College, Yangtze University, Hubei, Jingzhou 434023, China \\ Correspondence should be addressed to Zhang Jian; zhangjian0716@126.com
}

Received 6 April 2017; Revised 22 June 2017; Accepted 27 June 2017; Published 3 August 2017

Academic Editor: Somenath N. Sarkar

Copyright (c) 2017 Zhang Jian et al. This is an open access article distributed under the Creative Commons Attribution License, which permits unrestricted use, distribution, and reproduction in any medium, provided the original work is properly cited.

\begin{abstract}
In order to solve the problem that the measure precision of coal roof rock strain amount detection using traditional electric sensor is insufficient, a kind of High Sensitive Surface-Pasted Fiber Bragg Grating Strain Sensor based on rock bolting is designed for substitution. Furthermore, a kind of Reference Grating Zero-Strain method based on sensitivity matrix coefficient is proposed for solving FBG strain-temperature cross-sensitivity problem and realizing the better separation relevant to strain and temperature. It is proved that the sensitivity and measurement precision and anti-interference ability of FBG strain sensors are better than traditional electricity class sensors, being able to realize the real-time and online detection for coal roof surrounding rock strain.
\end{abstract}

\section{Preface}

Coal is the cornerstone of the national energy security and important basic industry related to the national economy. In recent years, strong demands appeared for coal quantity in the society, economy, and manufacture. The depth of coal excavation increases by $15-20 \mathrm{~m}$ annually. On the one hand it creates a lot of economic benefits. On the other hand coal mine power disasters have happened frequently because the natural deposit conditions of coal rock become more and more complex with deep mining. In particular, the disasters such as coal mine tunnel roof collapse and roof collapse bring about the major casualties with serious society influences.

For guaranteeing the safe operation of coal mining operations and the underground persons' safety, Detection and warning about coal mine roadway roof deformation are really essential and important. In recent years, a lot of wide and deep research jobs about detection means and prediction methods aimed at field and mining area collapse and coal mine roof and roof caving danger zone have appeared with plenty of investments coming from domestic and foreign government departments, major coal-producing enterprises, and universities. Foreign countries such as Australia and the United Kingdom use numerical simulation method to determine the rock support solution and the deformation monitor on the basis of experience analogy and theoretical analysis, including roadway geomechanical assessment, surrounding rock physical and mechanical parameters testing, the measurement of in situ stress magnitude and direction, site geological conditions survey, programming, and live performance monitoring [1]. The United States have drew up the warning regulation through measuring surrounding rock deformation state particularly. The domestic scholars Junmin et al. [2] proposed a kind of method of detecting sonicfrequency stress waves. Deshan and Qianwei [3] use wavelet domain analysis method based on ground penetrating radar to detect roof deformation in real time. Wei et al. [4] firstly tried to use electromagnetic radiation detection method. While all the above kinds of methods played a role in detecting in certain circumstances, however, because of the terrible environment under coal mine, higher requirements are needed about not only electromagnetic interference resistance and riot but also waterproof and moisture and cracking and corrosion resistance. Thus there exist great difficulties about the coal roof rock deformation detection and the reliability and service life of the instruments have been also 

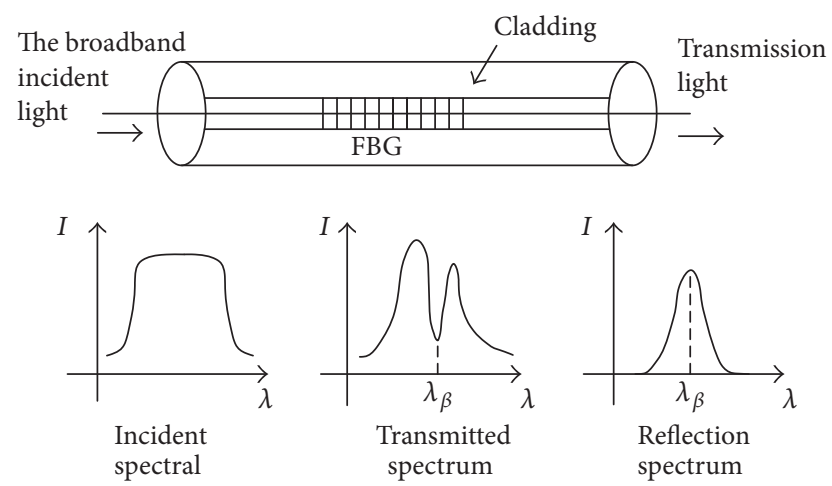

FIgURE 1: The structure schematic view of Fiber Bragg Grating.

affected directly. On the other hand, due to the complexity of disasters mechanism, including abundant influencing factors, sensors measurement requirements include long-distance, large space, distributed, and real-time online monitoring in time and space conditions. Hazard mechanism still can not be reflected using traditional sensing regulations and monitoring instruments completely now. Also it is difficult to meet the testing needs of the actual project.

Fiber optic sensing technique is an advanced sensing technique that has developed rapidly in recent years which has the advantages of light weight, electromagnetic interference resistance, high sensitivity, safety, reliability, corrosion resistance, distributed measurement, being easy to network, and plenty of other advantages. Comparing with the current detection technologies, it guaranteed the long-term online measurement accuracy and reliability improvement with expanding sensing measurement scope and variety conditions [5]. At the same time, it is able to adapt to special circumstances and requirements related to the coal mine roof rock strain detection compared to using traditional ways.

Firstly, cross-sensitivity issues were discussed for Fiber Bragg Grating coupling under thermal conditions of temperature and strain in this paper. Then a kind of specific surfacemounting Fiber Bragg Grating Strain Sensor based on bolting is designed and tested experimentally, while being applied to the particular coal mine roof rock strain detection. There are higher measurement precision and accuracy compared to traditional resistance-strain gauges way.

\section{Principle Analysis of Fiber Bragg Grating}

Sensing principle of fiber grating optical wavelength signal is shown in Figure 1: when a length of broadband light is incident to fiber grating, an optical wavelength that satisfied the Bragg condition will be reflected back to the grating and the light that does not satisfy the Bragg condition will be transmitted. In general, most of light will be transmitted and a small portion of light will be reflected. Then reflected or transmitted wavelength spectrum depends primarily on the period exchange amounts $\Delta \Lambda$ of the fiber grating and the effective refractive index of the fiber grating $n_{\text {eff }}$ based on Fiber Optic Grating coupled-mode theory. Fiber Bragg Grating wavelength will be drifted that caused any movement resulting in the two parameters' change. The relationship with the amount of wavelength change $\Delta \lambda_{B}[6]$ is

$$
\Delta \lambda_{B}=2 n_{\text {eff }} \Delta \Lambda .
$$

However, the effective refractive index $n_{\text {eff }}$ and the grating period variations $\Delta \Lambda$ will be affected by environmental factors (strain, temperature, etc.). When the Fiber Bragg Grating has been acted by stress and temperature and other external factors, due to the elastic action it will result in the transformation about the effective refractive index $n_{\text {eff }}$ and the grating period $\Lambda$, while the transformations related to these parameters will result in change in the reflection wavelength of the grating. Strain response model of temperature and resonance wavelength according to theoretical derivation can be expressed as follows:

$$
\frac{\Delta \lambda_{B}}{\lambda_{B}}=\left(1-P_{e}\right) \varepsilon+(\alpha+\xi) \Delta T,
$$

wherein $\varepsilon$ represents strain variation in the Fiber Bragg Grating; $P_{e}$ represents the fiber effective elastic optic coefficient; $\Delta T$ represents the temperature transformation. $\mu, \xi$, and $\alpha$ denote the Poisson coefficient of the fiber material, thermooptic coefficient, and thermal expansion coefficient.

Formula (2) shows that it also needed to analyze its strain sensing properties and temperature sensing performances when the Fiber Bragg Grating strain sensors are used to measure in the real environment.

\section{Research about Strain and Temperature Cross-Sensitive Issue}

In the field test of coal mine roadway roof, the cross-effects related to strain and temperature have existed at the same time and they will both act on the resonance wavelength. Therefore, wavelength transformation appearance that is motivated by strain or temperature can not be distinguished due to cross-effect [7]. In recent years, a series of solutions were proposed for solving Fiber Bragg Grating strain and temperature cross-sensitive issues, such as the adjacent double grating method [8], Long-period gratings and FBG bonding method [9], different cladding diameter fiber grating method [10], and birefringence distributed feedback (DFB) laser cavity method [11] from different applicable level; but these kinds of methods are not able to solve measurement accuracy problem about the Fiber Bragg Grating Strain Sensor used in coal mine tunnel roof rock deformation detection. For the measurement of Fiber Bragg Grating strain sensors applied to bolt supporting used in coal mine with harsh environmental conditions, this paper proposes a specific kind of reference zero-strain grating method based on sensitive coefficients matrix for separation about the strain and temperature. Therefore it can solve the strain and temperature cross-sensitivity issue preferably.

Starting from the equation $\lambda_{B}=2 n_{\text {eff }} \Lambda$, the resonance wavelength should be seen as function of two kinds of variate as temperature $T$ and strain $\varepsilon ; n=2 n_{\text {eff. }}$. Taylor series formula is used for expansion and when the variation ranges $\Delta \varepsilon$ and $\Delta T$ do not exceed the measurement range, order terms of $\Delta \varepsilon$ 


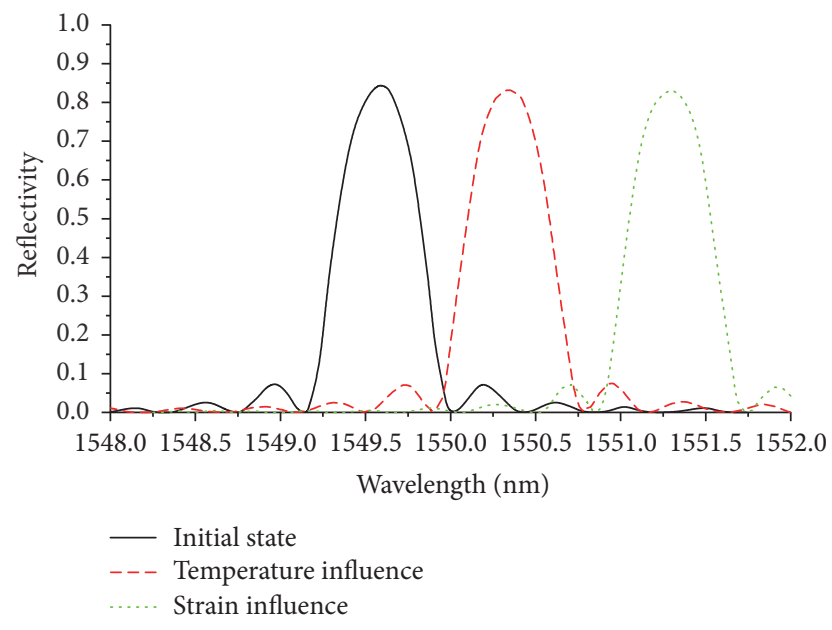

FIGURE 2: Fiber grating reflection spectrum with strain or temperature alone.

and $\Delta T$ compared with the lower order of $\Delta \varepsilon$ and $\Delta T$ terms can be ignored. Formula (3) is shown as follows:

$$
\Delta \lambda_{B}=K_{\varepsilon} \Delta \varepsilon+K_{T} \Delta T+K_{\varepsilon T} \Delta \varepsilon \Delta T .
$$

In order to solve the strain and temperature crosssensitivity problem, for formula (3), assuming that one ignores its cross-sensitivity items, then there is the formula shown as follows:

$$
\Delta \lambda_{B}=K_{\varepsilon} \Delta \varepsilon+K_{T} \Delta T .
$$

With formula (4) known, when strain and temperature of these two kinds of parameters are measured at the same time, the two parametric simultaneous equations can be used to calculate the size of strain and temperature. Setting up the Bragg grating wavelength shift transformation variation of two gratings as $\Delta \lambda_{1}$ and $\Delta \lambda_{2}$, respectively, the strain and temperature field are identical in the same external environment. The variation amount of the resonance wavelength expressed with matrix form is as follows:

$$
\left[\begin{array}{l}
\Delta \lambda_{1} \\
\Delta \lambda_{2}
\end{array}\right]=\left[\begin{array}{ll}
K_{1 \varepsilon} & K_{1 T} \\
K_{2 \varepsilon} & K_{2 T}
\end{array}\right]\left[\begin{array}{c}
\Delta \varepsilon \\
\Delta T
\end{array}\right]
$$

wherein $K_{1 \varepsilon}, K_{2 \varepsilon}$ represent each fiber grating strain sensitivity, respectively, and $K_{1 T}, K_{2 T}$ represent the corresponding temperature sensitivity, respectively.

By formula (5), in order to have a solution, the matrix must meet condition $\left[\begin{array}{ll}K_{1 \varepsilon} & K_{1 T} \\ K_{2 \varepsilon} & K_{2 T}\end{array}\right] \neq 0$ which requires that the characteristics of two Fiber Bragg Grating are different. Due to ignoring strain and temperature cross-sensitive items, the relative error analysis needs to be done.

As can be seen from Figures 2, 3, and 4, transformation range of strain and temperature is not large, so the relative error of the cross-sensitivity term is almost negligible. With the strain and temperature transformation increasing, the temperature cross-sensitive relative error is proportional to strain, while the relative error of temperature cross-sensitivity is also proportional to the strain changes.

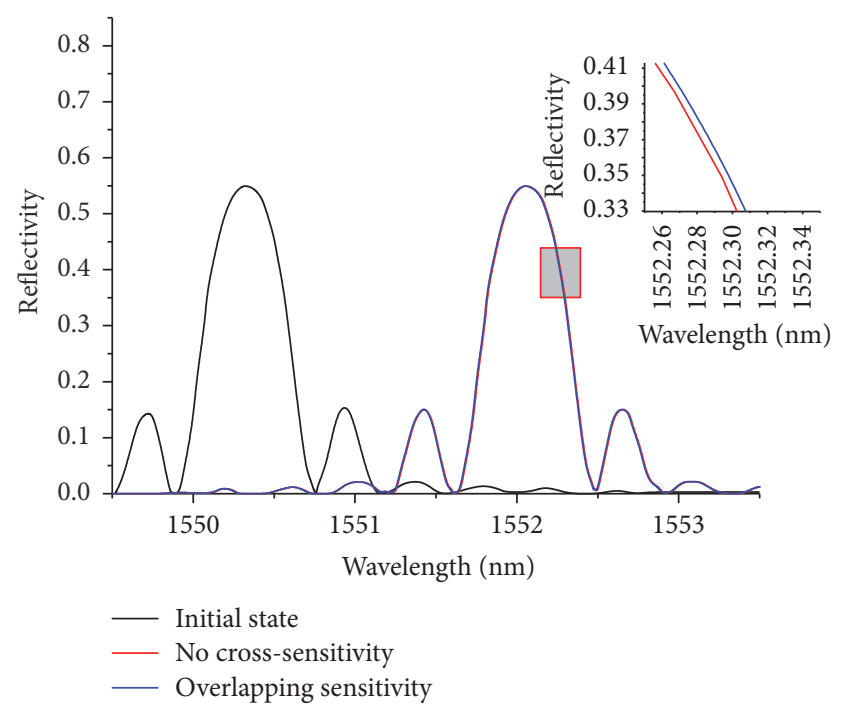

FIGURE 3: Fiber grating reflection spectrum comparison with or without strain and temperature cross-sensitivity-1.

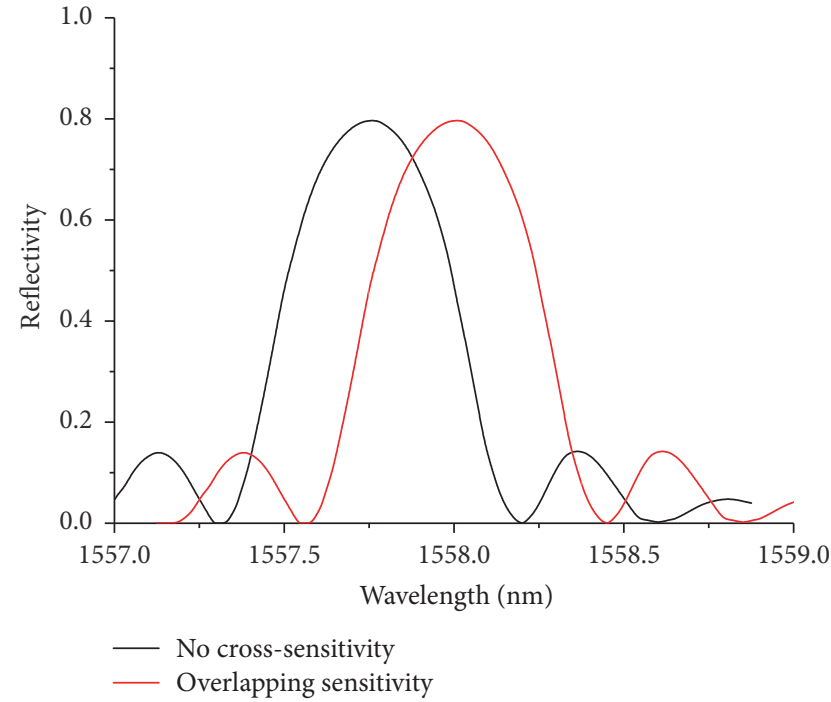

FIgURE 4: Fiber grating reflection spectrum comparison with or without strain and temperature cross-sensitivity-2.

According to the equation $\left[\begin{array}{l}\Delta \lambda_{1} \\ \Delta \lambda_{2}\end{array}\right]=\left[\begin{array}{ll}K_{12} & K_{1 T} \\ K_{2 \varepsilon} & K_{2 T}\end{array}\right]\left[\begin{array}{l}\Delta \varepsilon \\ \Delta T\end{array}\right]$, if one of the gratings in the similar environment is not affected by strain field but the temperature field, that is, $K_{2 \varepsilon}=0$, the Bragg resonance wavelengths of two gratings are different, and formula (5) can be rewritten as the following formula:

$$
\left[\begin{array}{l}
\Delta \lambda_{1} \\
\Delta \lambda_{2}
\end{array}\right]=\left[\begin{array}{cc}
K_{1 \varepsilon} & K_{1 T} \\
0 & K_{2 T}
\end{array}\right]\left[\begin{array}{c}
\Delta \varepsilon \\
\Delta T
\end{array}\right]
$$

In formula (6), $\Delta \lambda_{1}$ represents the wavelength drift of the one fiber grating under the effect of strain and temperature simultaneously, while $\Delta \lambda_{2}$ represents the wavelength drift of another fiber grating under the effect of temperature field.

For the two gratings, $K_{1 \varepsilon} \neq 0, K_{1 T} \neq 0$, and $K_{2 T} \neq 0$ can be obtained. That is, $\left[\begin{array}{ccc}K_{18} & K_{1 T} \\ 0 & K_{2 T}\end{array}\right] \neq 0$. Therefore the equation 


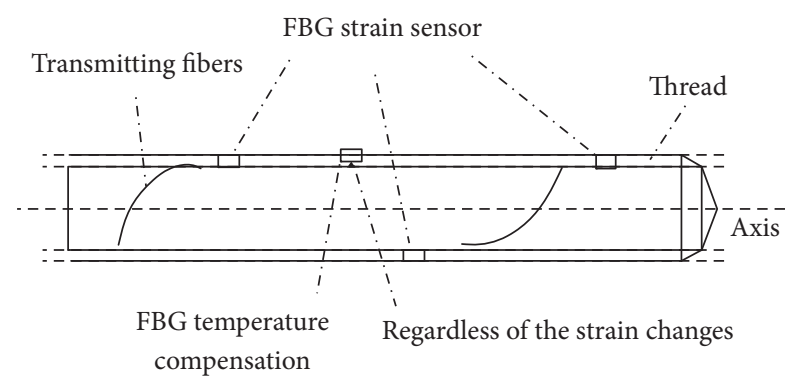

FIGURE 5: Strain sensor layout schematic.

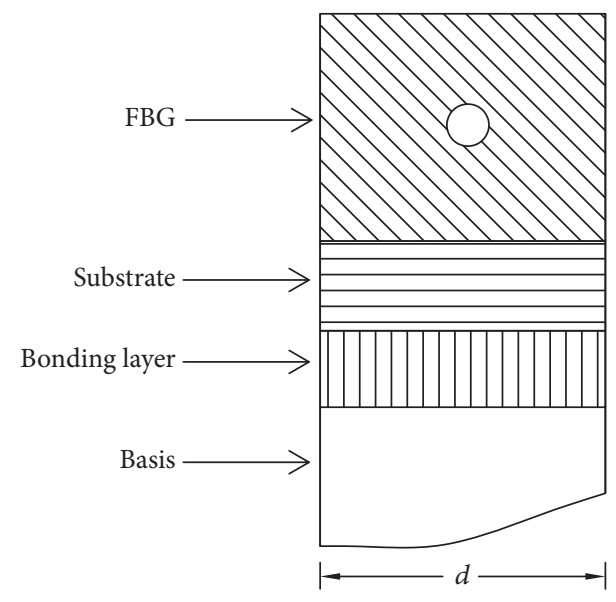

FIGURE 6: Fiber grating adhered to the cross-section of the bolt.

must offer solution and thus $\Delta \varepsilon$ and $\Delta T$ can be obtained. That is, the simultaneous detection related to the strain and temperature can be realized by measuring both of the grating wavelength shifts.

\section{Layout and Strain Transfer Analysis of the Fiber Bragg Grating Strain Sensor}

The internal stress that existed in coal mine underground roadway is an essential force causing rock deformation and breakage. Therefore the roof security situation is reflected through online testing aimed at the stress-strain of the coal mine roadway roof. The Fiber Bragg Grating strain sensors that are designed in this paper evaluate the security situation through detecting support anchors roof rock stress effect.

Layout distribution of the Fiber Bragg Grating strain sensors are shown as Figure 5. Sensors were laid along the axial direction of anchor rod, and three Fiber Bragg Grating strain sensors were pasted on each bolt and cascaded through a transmission fiber. Taking the cross-sensitivity issue into account, zero-strain temperature sensor needs to be set up that was unaffected by axial stress and separated strain and temperature.

Figure 6 is a cross-sectional view of fiber grating affixed on the bolt. It can be seen through the figure structure that there appeared a backing layer, an adhesive layer, and others between fiber gratings and anchor rod after pasting.
Comparing the elastic modulus of these materials with the elastic modulus of the fiber is quite different. Therefore it will cause the error between the measured strain value and actual value of the strain suffered rod. So it is necessary to establish the corresponding mechanical model between Fiber Bragg Grating strain sensors that have been pasted and the anchor rod for analyzing the strain transformation rules.

Figure 7 is the strain transfer analysis chart about bonded FBG sensors and the anchor rod. In this figure $h_{p}$ shows the thickness of the upper adhesive layer, $h_{b}$ shows the thickness of the substrate, $h_{a}$ shows the thickness of the lower portion of the adhesive layer, the radius of the fiber grating is set up $r_{g}$, width is set up $d$, at the same time, $\tau_{p g}, \tau_{p b}, \tau_{b a}$, and $\tau_{a h}$ represent respective shear force between each adjacent layer, bonded length is set to $2 L$, and $d \sigma_{g}, d \sigma_{p}, d \sigma_{b}$, and $d \sigma_{a}$ are axial stress of the fiber grating, the upper portion of the adhesive layer, the substrate, and the lower adhesive layer, respectively.

Strain transfer distribution coefficients are shown as the following formula:

$$
T(z)=\frac{\varepsilon_{g}(z)}{\varepsilon_{h}}=1-\frac{\sinh (k z)}{k L \cosh (k L)} .
$$

$E_{g}=70 \mathrm{Gpa}, r_{g}=6.25 \mathrm{~mm}, G_{b}=1.34 \mathrm{Gpa}, G_{a}=$ $0.01 \mathrm{Gpa}, h_{p}=12.5 \mathrm{~mm}, h_{a}=2.5 \mathrm{~mm}$, and $2 L=40 \mathrm{~mm}$ have been set. On the basis of these classical parameters configuration, the size of $G_{a}, h_{a}$, and $L$ will be changed, respectively. This paper simulation analyzes the strain transfer distribution coefficients. The simulation results are shown in Figures 8, 9, and 10 .

As can be seen from Figures 8, 9, and 10, for the usage of surface-pasted way, if the thickness of the adhesive layer is larger, then the transfer efficiency is worse. This means that the size of the adhesive layer thickness will affect the strain transfer performance tremendously; meanwhile, the bonding length of the fiber gratings will also affect the strain transfer performance greatly. As can be seen from the simulation chart, when the bonding length $L$ is comparatively short, the grating strain transfer effect that passed from anchor rod is poor. Therefore, in terms of certain length of grating, strain variation of each part is different, so that the measurement accuracy of the fiber gratings and other indicators will be affected completely. It follows that higher transfer coefficient should be got, shear modulus of the adhesive layer must be improved, and the thickness of the adhesive layer must be reduced. In addition, for the bonding length that is long enough, the middle grating section of the whole optical fiber can percept the strain transmission of the anchor rod completely.

\section{Strain Test Analysis of the Fiber Bragg Grating Strain Sensor}

Under the same pressure, the axial strain caused by transverse stress of grating is much smaller than the axial strain caused by axial stress. Comparing with the wavelength drift of the reflected light caused by axial strain under the similar parameters, the sensitivity of transverse stress is low. If the 

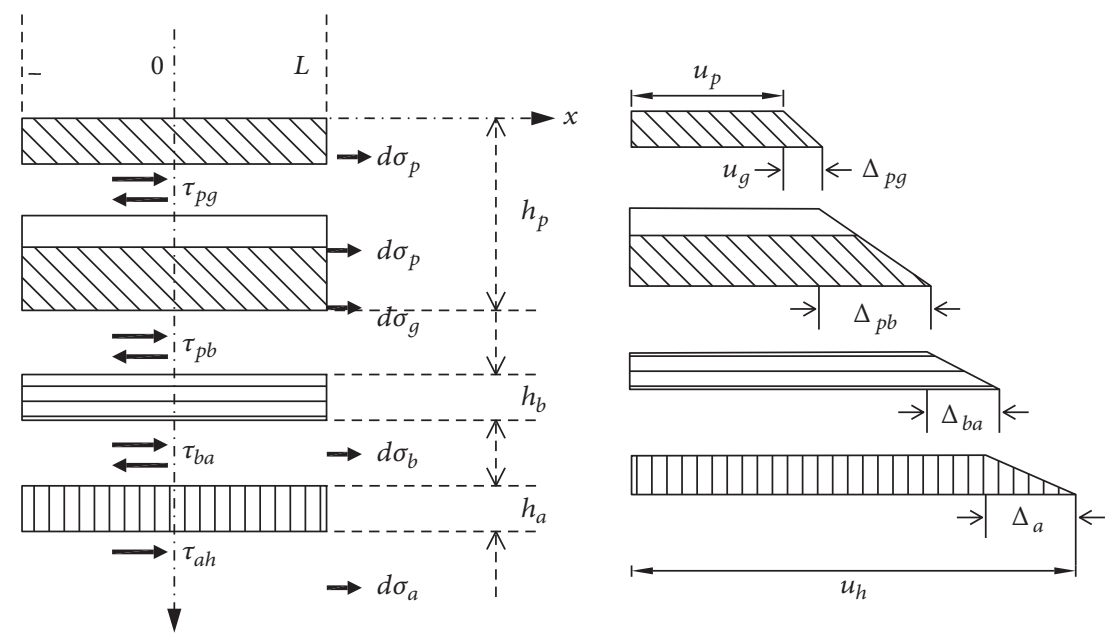

FIGURE 7: FBG sensing strain transfer analysis chart.

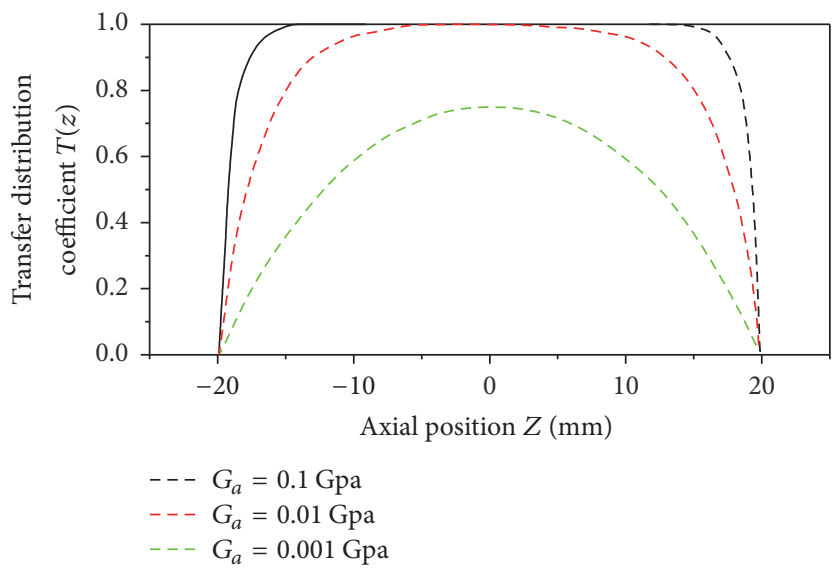

FIGURE 8: Effect of $G_{a}$ on the transfer coefficient.

force situation is complicated, then the wavelength shift caused by the axial strain will play a decisive role.

5.1. Resistance-Strain Gauges Test of Bolt. Firstly, tensile deformation tests will be made 12 times aim at anchor rod using resistance-strain gauges under different tensile load conditions, as shown in Table 1.

The experiment data of Table 1 processed graphically are shown in Figure 11. The strain curve analysis shows that the overall distribution of the transformation in the strain is uniform. With the gradual increase of the tensile load, strain curves show a gradual increasing transform trend.

After the data are graphically processed in Table 1, each resistance-strain gauge strain curve with increasing load is shown in Figure 12.

As can be seen from Figure 12, at the position near the bolt end, the curve slope of the resistance-strain gauge 1 is larger than others. The curve slope of the resistance-strain gauge 2 is smaller than the curve slope of the resistance-strain gauge 1 . But it can still reflect the strain transformation of anchor rod clearly. Because the slope of resistance-strain gauge 3 near the

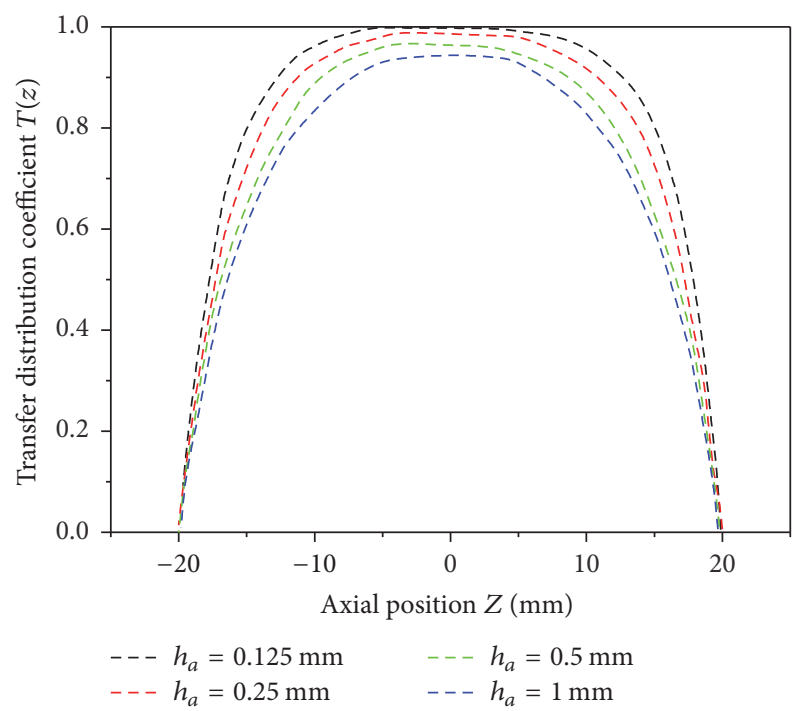

FIGURE 9: Effect of $h_{a}$ on the transfer coefficient.

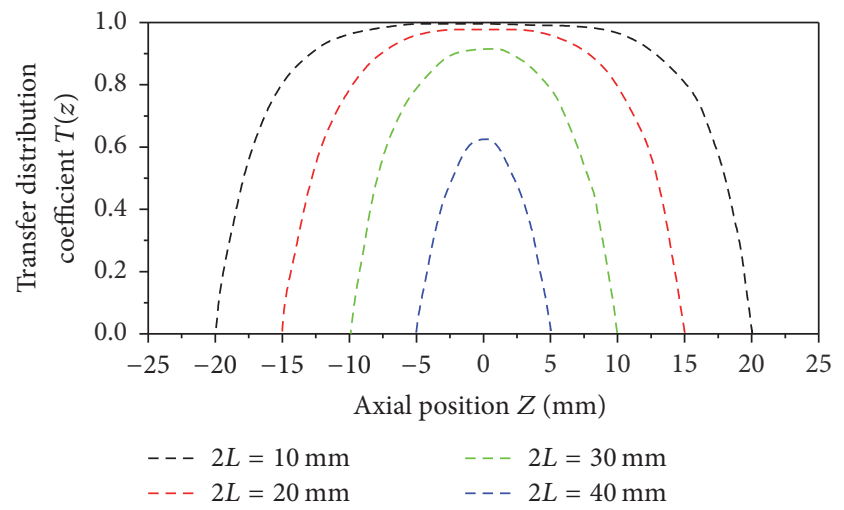

Figure 10: Effect of $L$ on the transfer coefficient.

tail bolt end is small, this means that the descriptions match the theoretical analysis of the bolt stress. 
TABLE 1: Resistance strain gauges experimental data under different tensile load.

\begin{tabular}{|c|c|c|c|c|c|c|}
\hline \multirow{2}{*}{ Load times } & \multicolumn{2}{|c|}{ Load } & \multirow[b]{2}{*}{ Load times } & \multicolumn{3}{|c|}{ Resistance strain gauge measurements/ $\mu \varepsilon$} \\
\hline & Load/kN & Tension/MPa & & $\begin{array}{c}\text { Resistance strain } \\
\text { gauges } 1\end{array}$ & $\begin{array}{c}\text { Resistance strain } \\
\text { gauges } 2\end{array}$ & $\begin{array}{c}\text { Resistance strain } \\
\text { gauges } 3\end{array}$ \\
\hline 0 & 0 & 0 & 14:01:21 & 0 & 0 & 0 \\
\hline 1 & 6.51 & 2.20 & $14: 01: 54$ & 223 & 155 & 8 \\
\hline 2 & 13.03 & 4.41 & 14:02:31 & 287 & 243 & 9 \\
\hline 3 & 19.55 & 6.62 & 14:02:52 & 402 & 360 & 11 \\
\hline 4 & 26.09 & 8.82 & 14:03:14 & 547 & 476 & 16 \\
\hline 5 & 32.61 & 10.05 & 14:03:50 & 630 & 563 & 19 \\
\hline 6 & 39.13 & 13.25 & 14:04:01 & 717 & 615 & 23 \\
\hline 7 & 45.70 & 15.52 & $14: 04: 28$ & 824 & 725 & 26 \\
\hline 8 & 52.23 & 17.75 & $14: 04: 48$ & 934 & 795 & 29 \\
\hline 9 & 58.59 & 19 & 14:05:05 & 1028 & 887 & 33 \\
\hline 10 & 64.66 & 21 & $14: 05: 34$ & 1143 & 1008 & 37 \\
\hline 11 & 70.54 & 23 & 14:05:55 & 1224 & 1133 & 40 \\
\hline 12 & 76.42 & 24 & $14: 06: 21$ & 1325 & 1263 & 44 \\
\hline
\end{tabular}

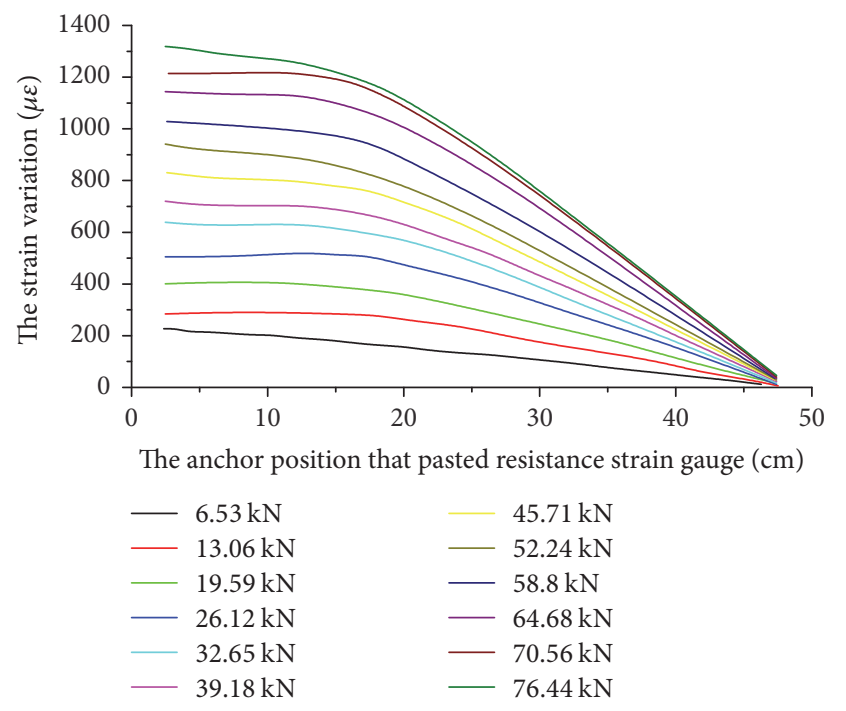

FIGURE 11: Bolt strain curve detected with resistance-strain gauges.

5.2. Test of Bolt Fiber Bragg Grating Strain. Fiber Bragg Grating (FBG) strain sensors have been laid at the adjacent position of resistance-strain gauge. Since the temperature difference of the experimental environment is little, therefore only one reference Fiber Bragg Grating temperature sensor has been used. Because the fiber grating wavelength transfer instantaneous value has been recorded with each load, Fiber Bragg Grating wavelength drift value can be calculated through this value subtracting the initial wavelength of each load. The test data of grating strain are shown in Table 2. Due to the separation requirement related to temperature and strain cross-sensitivity, this means that the wavelength drift value of temperature sensor (FBG4) can be got through reference zero-strain grating method according to the wavelength drift values of FBG1, FBG2, and FBG3. Then wavelength drift

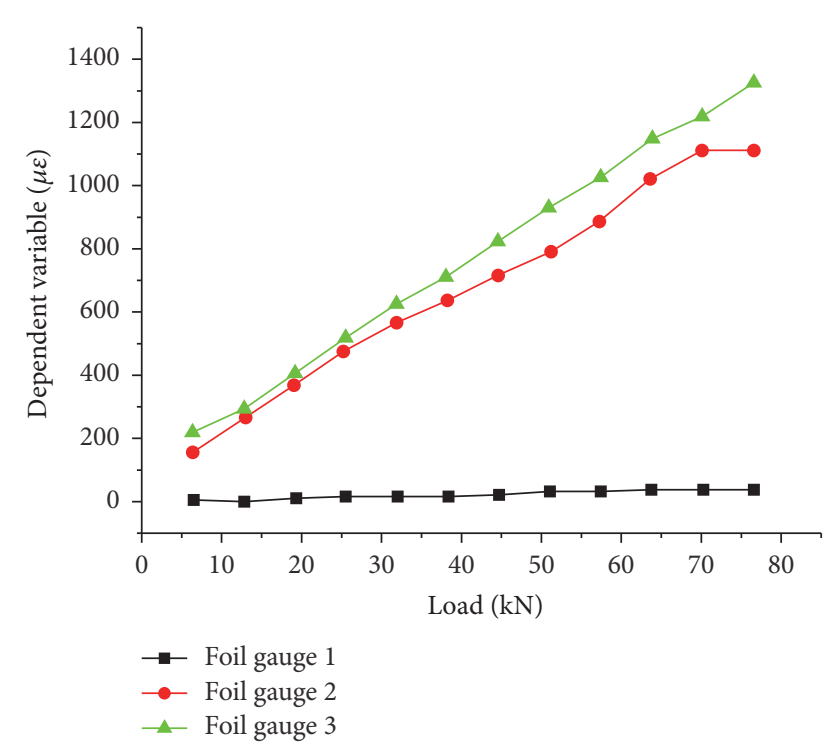

FIGURE 12: The relationship diagram between strain of resistance gauges and load.

value of the fiber grating will be got caused by bolt deformation. After the above process, the relationship between Fiber Bragg Grating wavelength sensitivity coefficients and axial strain of fiber grating is about $1.205 \mathrm{pm} / \mu \varepsilon$ based on the data in Table 2. At the same time, the load strain variation related to corresponding detection points can be calculated and shown in Table 2, wherein Poisson's ratio coefficient is $\mu \approx 0.17, p_{11} \approx 0.113$, and $p_{12} \approx 0.252$. The core refractive index of the germanium doped fiber is $n_{\text {eff }} \approx 1.46$. Thus the elastic coefficients have been obtained as $P_{e} \approx 0.217$, $\zeta \approx 7.0 \times 10^{-6} /{ }^{\circ} \mathrm{C}$, and $a \approx 0.5 \times 10^{-6} /{ }^{\circ} \mathrm{C}$, selecting Bragg center wavelength as $\lambda_{0}=1550 \mathrm{~nm}$.

According to $K_{\varepsilon}=d \lambda / d \varepsilon=\lambda_{0}\left(1+\left(1 / n_{\text {eff }}\right)\left(\partial n_{\text {eff }} / \partial \varepsilon\right)\right)=$ $\lambda_{0}\left\{1-\left(n_{\text {eff }}^{2} / 2\right)\left[p_{12}-\mu\left(p_{11}+p_{12}\right)\right]\right\}$, the axial sensitivity 
TABLE 2: Data of FBG strain test.

\begin{tabular}{lcccccc}
\hline \multirow{2}{*}{ Load times } & \multicolumn{2}{c}{ Load } & & \multicolumn{2}{c}{ Fiber Bragg Grating Sensor Wavelength shift/pm } & \multicolumn{2}{c}{ Temperature } \\
compensation/pm
\end{tabular}

TABLE 3: Data of FBG tensile strain test.

\begin{tabular}{|c|c|c|c|c|c|}
\hline \multirow{2}{*}{ Load times } & \multicolumn{2}{|c|}{ Load } & \multicolumn{3}{|c|}{ Fiber Bragg Grating strain detected/ $\mu \varepsilon$} \\
\hline & Load/kN & Tension/MPa & FBG1 & FBG2 & FBG3 \\
\hline 0 & 0 & 0 & 0 & 0 & 0 \\
\hline 1 & 6.53 & 2.22 & 165 & 106 & 5 \\
\hline 2 & 13.06 & 4.44 & 235 & 196 & 8 \\
\hline 3 & 19.59 & 6.66 & 347 & 290 & 12 \\
\hline 4 & 26.12 & 8.88 & 451 & 413 & 15 \\
\hline 5 & 32.65 & 11.1 & 573 & 506 & 18 \\
\hline 6 & 39.18 & 13.32 & 650 & 560 & 22 \\
\hline 7 & 45.71 & 15.54 & 761 & 669 & 25 \\
\hline 8 & 52.24 & 17.76 & 875 & 734 & 28 \\
\hline 9 & 58.8 & 20 & 970 & 836 & 32 \\
\hline 10 & 64.68 & 22 & 1082 & 951 & 37 \\
\hline 11 & 70.56 & 24 & 1164 & 1070 & 41 \\
\hline 12 & 76.44 & 26 & 1264 & 1206 & 44 \\
\hline
\end{tabular}

coefficients can be calculated as $K_{\varepsilon}=1.205 \mathrm{pm} / \mu \varepsilon$, and $K_{T}=$ $9.95 \mathrm{pm} /{ }^{\circ} \mathrm{C}$.

According to $K_{T}=d \lambda / d T=\lambda_{B}\left(a+\left(1 / n_{\text {eff }}\right)\left(\partial n_{\text {eff }} / \partial T\right)\right)=$ $\lambda_{B}(a+\zeta)$, the temperature sensitivity coefficients can be calculated as $K_{T}=9.95 \mathrm{pm} /{ }^{\circ} \mathrm{C}$.

According to another Reference Grating ZeroStrain equation based on sensitivity coefficient $\left[\begin{array}{l}\Delta \lambda_{1} \\ \Delta \lambda_{2}\end{array}\right]=$ $\left[\begin{array}{cc}K_{1 \varepsilon} & K_{1 T} \\ 0 & K_{2 T}\end{array}\right]\left[\begin{array}{c}\Delta \varepsilon \\ \Delta T\end{array}\right]$, the Fiber Bragg Grating strain variations can be calculated and shown in Table 3 , wherein $\Delta \lambda_{2}$ is the wavelength shift of temperature compensation-FBG4.

Then the experimental data from Table 3 are processed graphically. Bolt strain curves that are detected by Fiber Bragg Grating strain sensors placed on different positions are shown as Figure 13.

As can be seen from Figure 13, the overall strain distributions detected by Fiber Bragg Grating strain sensors under different loads conditions are uniform, which are similar to the measurement results of the resistance-strain gauges. The strain in the end of the bolt will become large. As the load continues to increase, strain becomes larger trend progressively than before. After the experimental data of Table 3 are processed graphically, the corresponding strain curve of each of the Fiber Bragg Grating load increases is shown in Figure 14. As can be seen from the figure, the linear transformation trend is better than the development trend using resistance-strain gauges.

5.3. Analysis of Test Results. Force theoretical equation aimed at anchor rod carried load loading is as follows:

$$
N=P \exp \left(-\frac{t z^{2}}{2}\right) .
$$




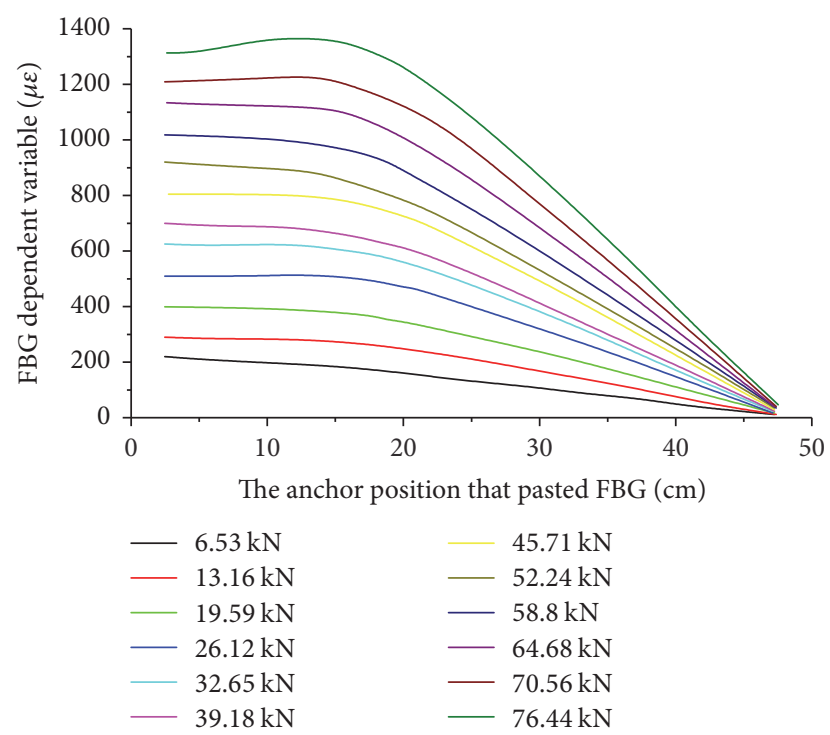

FIGURE 13: FBG Bolt strain curve.

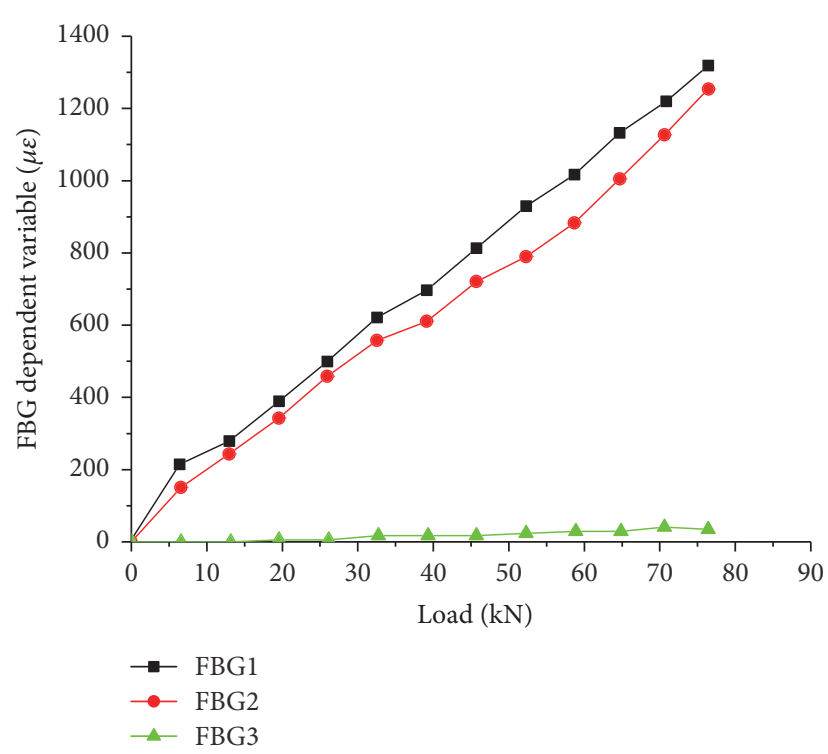

FIGURE 14: Fiber Bragg Grating strain and load diagram.

When the load tension reaches $76.44 \mathrm{kN}, t=7.83$ can be obtained by the equipment parameters test, and the theoretical value of bolt axial force in this case is

$$
N=76.44 \cdot e^{-3.91 z^{2}}
$$

When the load reaches $76.44 \mathrm{KN}$, the theoretical value of resistance-strain gauge and the Fiber Bragg Grating axial force distribution trends are shown in Figure 15. These threeaxis force distribution curves do not coincide. Therefore it is necessary to do error analysis of the load loading test aimed at anchor rod.

Firstly, strain transformation of the test is analyzed. Due to the related factors effect about test equipment, the problem of strain transfer efficiency must exist. In order to prevent the

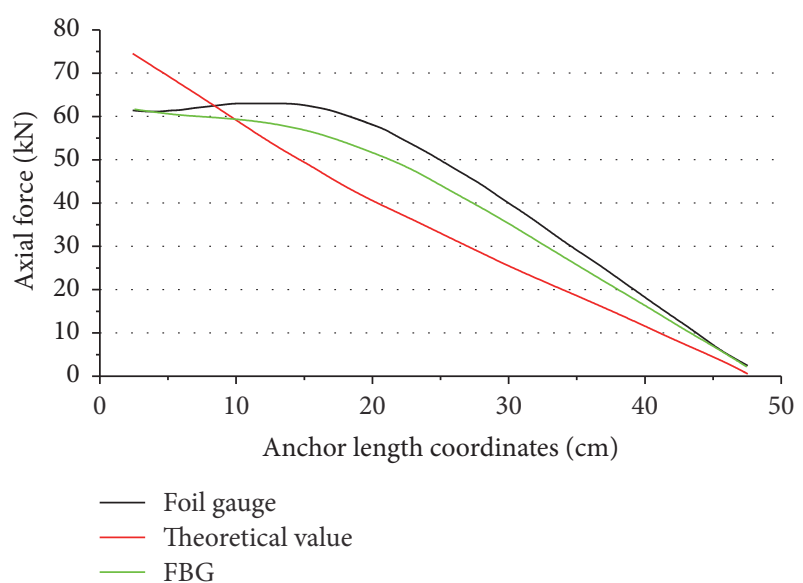

FIGURE 15: Axial force distribution of resistance-strain gauge and the theoretical value and fiber grating.

test and calculation error from the modulus parameters configuration, this paper analyzes strain transformation based on the strain of resistance-strain gauge. According to the strain datasheet of resistance-strain gauge and the strain datasheet of Fiber Bragg Grating, the transfer coefficient of Fiber Bragg Grating can be calculated with respect to the resistance-strain gauge strain data, which is

$$
\beta=\frac{\varepsilon_{g}}{\varepsilon_{R}}
$$

Calculating through formula (10), corresponding each transfer coefficient average values of each Fiber Bragg Grating with respect to strain data of the resistance-strain gauges are $\beta_{1}=0.894, \beta_{2}=0.875$, and $\beta_{3}=0.867$. The average value of average transfer coefficients related to three measuring points according to corresponding each fiber grating can be calculated as $\bar{\beta}=0.879$. While the strain transfer coefficient related to resistance-strain body is less than 0.879 , there have been some errors between results using fiber grating measurement and the oretical values.

Secondly, through formulas (8) and (9) the axial force fitting formula can be set as:

$$
N=P \cdot e^{-k x^{2}}
$$

In which $k$ is a proportionality factor. Bolt test data with the load is $76.44 \mathrm{kN}$, just as $p=76.44$, When three measurement values fit into equation (11) and coefficient $k$ can be obtained, Thus the fitted axial force formulas obtained by Fiber Bragg Grating and resistance-strain gauges are:

$$
\begin{aligned}
& N_{g}=76.44 \cdot e^{-2.71 z^{2}} \\
& N_{R}=76.44 \cdot e^{-2.58 z^{2}}
\end{aligned}
$$

It can be seen from Figure 15, three-axis force distribution curves of resistance-strain gauges and Fiber Bragg Grating and theoretical values are within the same axial range, the 
relative error of resistance-strain gauge and Fiber Bragg Grating can be calculated as

$$
\begin{aligned}
\frac{\delta N_{g}}{N} & =\frac{\int_{0}^{50} 76.44 \cdot e^{-2.71 z^{2}} d z-\int_{0}^{50} 76.44 \cdot e^{-3.91 z^{2}} d z}{\int_{0}^{50} 76.44 \cdot e^{-2.71 z^{2}} d z} \\
& =0.0767 \\
\frac{\delta N_{R}}{N} & =\frac{\int_{0}^{50} 76.44 \cdot e^{-2.58 z^{2}} d z-\int_{0}^{50} 76.44 \cdot e^{-3.91 z^{2}} d z}{\int_{0}^{50} 76.44 \cdot e^{-2.58 z^{2}} d z} \\
& =0.0984 .
\end{aligned}
$$

Error analysis illustrates that the measurement relative error of the resistance-strain gauge is $9.84 \%$ and measurement relative error of Fiber Bragg Grating is 7.67\%. Not only the test and measurement operation but also Fiber Bragg Grating paste process is the main reason that causes errors.

\section{Conclusion}

This paper has solved the problem about coal mine tunnel roof rock deformation security detection and designed a kind of surface-mounting Fiber Bragg Grating Strain Sensor based on bolting which applied Reference Grating Zero-Strain way using sensitivity matrix coefficients. Thus it can overcome the strain and temperature cross-sensitivity issue related to the fiber grating effectively. The Fiber Bragg Grating sensors have higher measurement accuracy and sensitivity and strong anti-interference compared to traditional electrical sensors. When comparing with the normal electrical sensors, the strain variation of the coal mine roof rock will be detected more accurately than before. That is important meaning of protecting underground work safety.

\section{Conflicts of Interest}

The authors declare that there are no conflicts of interest regarding the publication of this paper.

\section{Acknowledgments}

This work is supported by Educational Commission of Hubei Province of China under Grant no. B2015445; supported by Humanities and Social Science Foundation of Yangtze University, no. 2015csy002; supported by Chu Culture Research Institute Open Foundation Project of Yangtze University in 2017; supported by the Humanities and Social Science Foundation of Hubei Provincial Department of Education, Youth Foundation, Yangtze University, 17Q057; supported by College Level Teaching Research General Project of Yangtze University, no. JY2015037; and supported by the Humanities and Social Science Research Project of Jingzhou Think Tank in 2017, no. Jzsk-yk1734.

\section{References}

[1] L. Dou, C. Lu, Z. Mu, and M. Gao, "Prevention and forecasting of rock burst hazards in coal mines," Mining Science \& Technology, vol. 19, no. 5, pp. 585-591, 2009.

[2] W. Junming, C. Yiqun, and C. Hua, "Expressway anchor bar quality nondestructive testing technology," Progress in Geophysics, vol. 04, pp. 782-785, 2004.

[3] F. Deshan and D. Qianwei, "Ground penetrating radar wavelet domain three- dimensional wave equation migration," Chinese Journal of Geophysics, vol. 02, pp. 566-574, 2008.

[4] M.-Y. Wei, E.-Y. Wang, X.-F. Liu, and C. Wang, "Numerical simulation of rockburst prevention effect by blasting pressure relief in deep coal seam," Rock and Soil Mechanics, vol. 32, no. 8, pp. 2539-2560, 2011 (Chinese).

[5] L. Chuan and M. Yi, Fiber Grating: Principles, Technology and Sensing Applications, Beijing Science Press, Beijing, China, 2005.

[6] L. Yanbiao, Fiber Optics, Tsinghua University Press, Beijing, China, 2000.

[7] W. Zhang, Q. Tu, S. Lei, G. Kai, S. Yuan, and X. Dong, "Recent progress in theory fbg sensors, design and application," Progress in Physics, vol. 04, 2004.

[8] Z. Shen, J. Zhao, and X. Zhang, "Fiber Grating Fabry - Perot sensor Frequency Division Multiplexing," Optics, vol. 07, pp. 1173-1177, 2007.

[9] Y. Miao, B. Liu, and Q. Zhao, "Different cladding diameter tilted fiber grating refractive index sensing properties," Optics, vol. 11, pp. 2072-2076, 2008.

[10] B. Shuai, L. Xia, Y. Zhang, C. Zhou, and D. Liu, "Principle and progress of surface plasmon resonance sensor based on grating," Laser \& Optoelectronics Progress, vol. 10, pp. 5-15, 2011.

[11] J. Yuan, X. Dong, and L. Wang, "Randomly distributed string feedback erbium-doped fiber laser based on weak reflection FBG," Optoelectronics \& Laser, vol. 1, no. 1, pp. 21-26, 2015. 


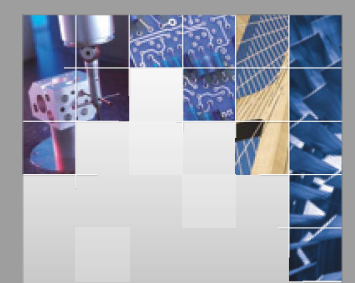

\section{Enfincering}
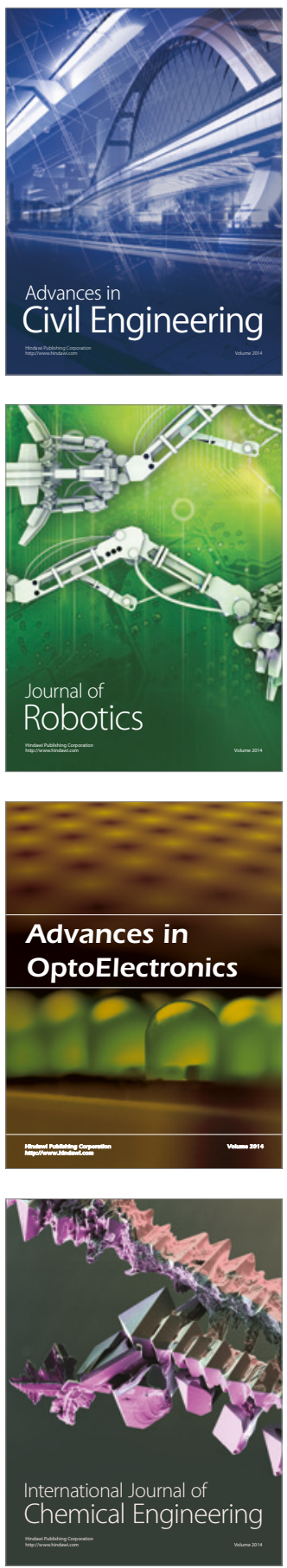

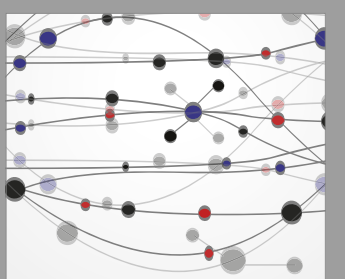

The Scientific World Journal

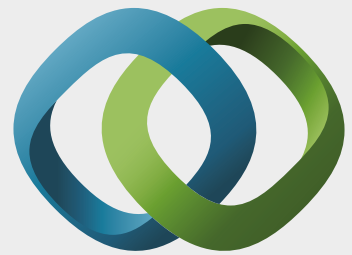

\section{Hindawi}

Submit your manuscripts at

https://www.hindawi.com
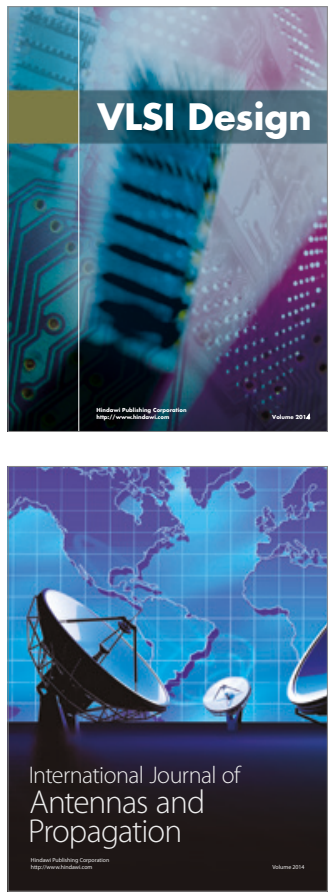

\section{Rotating}

Machinery
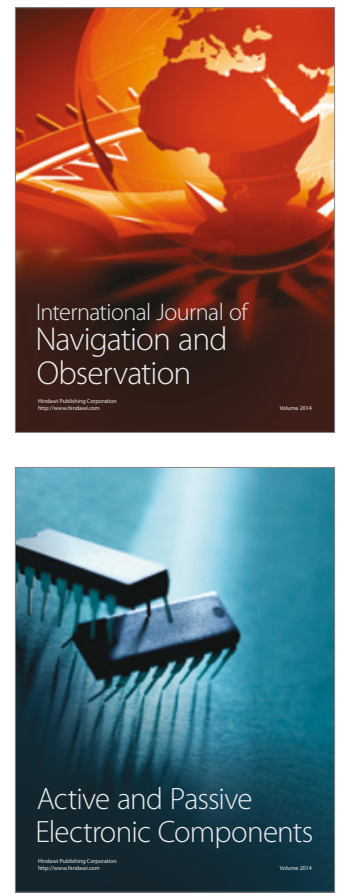
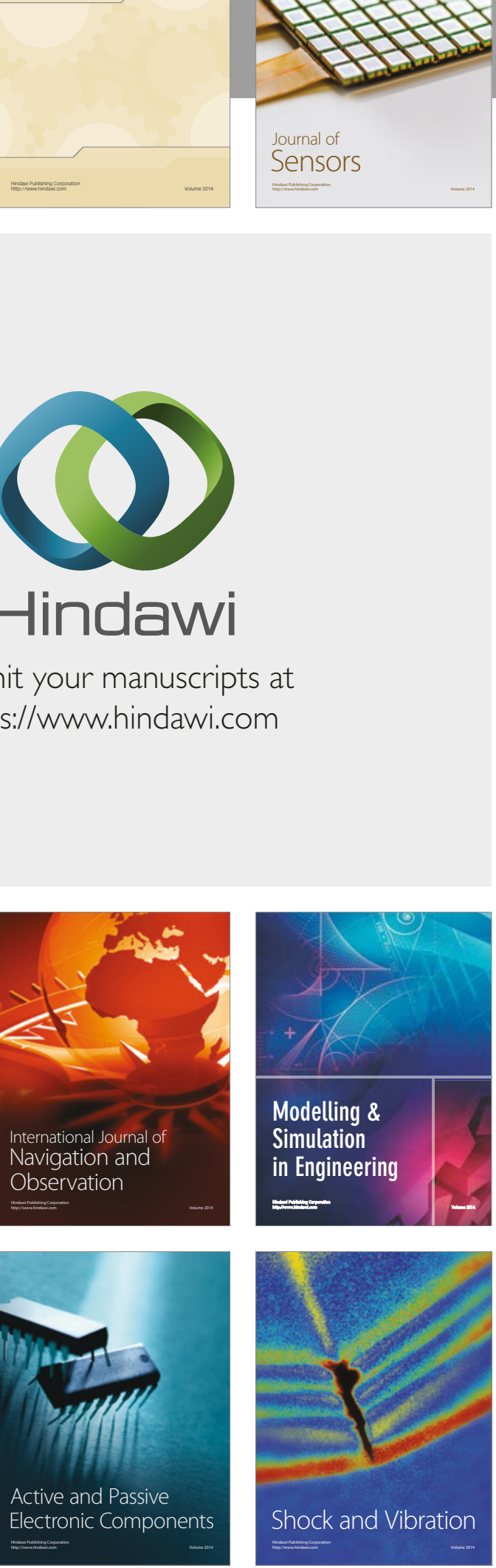
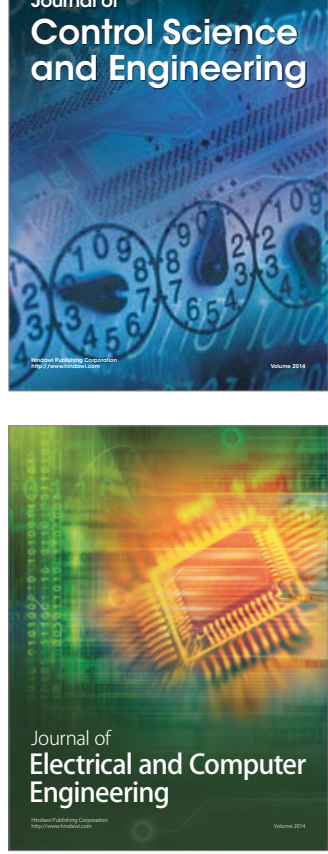

Distributed

Journal of

Control Science

and Engineering
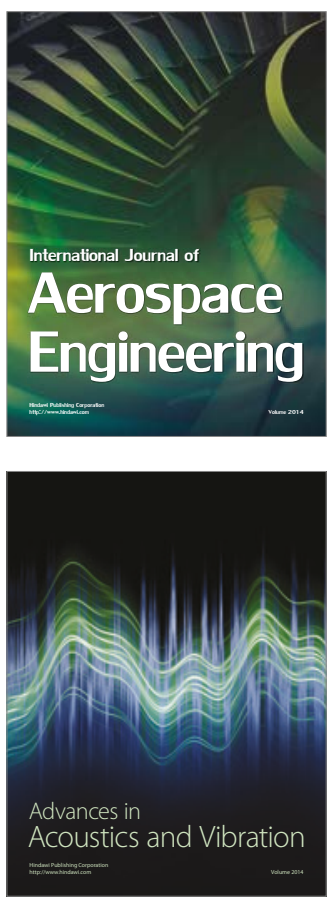

Sensor Networks 